\title{
Principios del uso de TIC's para proyectos productivos en instituciones educativas rurales
}

\section{Principles of the Use of ICT's for productive projects in rural educational institutions}

\author{
Carlos Ramon Vidal Tovar ${ }^{a, b 1}$ (D), Carlos Julio Lattá Arias ${ }^{c(\mathbb{D})}$ y Carlos \\ Alberto Severiche Sierra ${ }^{d(D)}$ \\ ${ }^{a}$ Grupo de Investigación CRECI, Universidad Popular del Cesar. \\ Valledupar, Cesar, Colombia \\ ${ }^{b}$ Grupo de Investigación CIEMPIES, Universidad del Santander \\ -UDES. Valledupar, Cesar, Colombia \\ ${ }^{c}$ I.E.D Las Mercedes, Secretaria de Educación Departamental del \\ Magdalena, Zona Bananera, Magdalena, Colombia \\ ${ }^{d}$ Grupo de Investigación GASST, Corporación Universitaria Minuto \\ de Dios - UNIMINUTO, Barranquilla, Atlantico, Colombia
}

\begin{abstract}
El objetivo fue identificar los principios del Uso de las TIC's para proyectos productivos en las instituciones educativas del Municipio Zona Bananera. Magdalena, Colombia. La investigación es descriptiva, el diseño es de campo, no experimental transaccional. La población estuvo conformada por directivos y docentes 12 Instituciones Educativas de básica media, donde se estudió a rectores y personal docente. La técnica de recolección de datos utilizada consiste en la observación mediante encuesta, como instrumento de recolección de datos se emplea un cuestionario bajo una escala tipo Likert. Como conclusión, los directivos y docentes con poca presencia identifican los principios del uso de las TIC's; Responsabilidad, corresponsabilidad y participación protagónica, por lo cual no son considerados en las instituciones educativas para elevar la capacidad productiva en el desarrollo de proyectos productivos.
\end{abstract}

Palabras Claves. Informática educativa; Investigación formativa; Tecnologías de la información y la comunicación; Zona rural.

\begin{abstract}
The objective was to identify the principles of the use of ICT's for productive projects in educational institutions of the Municipality of the Zona Bananera. Magdalena, Colombia. Research is descriptive, design is field, not transactional experimental. The population was made up of managers and teachers 12 elementary schools, where principals and teaching staff were studied. The data collection technique used consists of observation through a survey, as a data collection instrument a questionnaire is used under a Likert-type scale. In conclusion, managers and teachers with little presence identify the principles of the use of ICT's; Responsibility, co-responsibility and leading participation, which is why they are not considered in educational institutions to increase productive capacity in the development of productive projects.
\end{abstract}

1 e-mail: carlosvidal@unicesar.edu.co 
Keywords. Educational informaTIC's; Formative research; Information and communication technologies; Rural area.

Como Citar. C. R. Vidal Tovar, C. J. Lattá Arias y C. A. Severiche Sierra, 'Principios del uso de TIC's para proyectos productivos en instituciones educativas rurales", Jou. Cie. Ing., vol. 12, no. 1, pp. 110-115, 2020. doi: $10.46571 / \mathrm{JCI} .2020 .1 .9$

\section{Recibido: 05/04/2020 Revisado: 18/05/2020 Aceptado: 22/07/2020}

\section{Introducción}

Las tecnologías de la información y comunicación, denominadas TIC's, han arropado a todas las actividades realizadas por los seres humanos, desde las de orden cotidiana, hasta las de índole laboral, científico, deportivas, culturales, lo que ha conllevado a capacitarse en ese sentido, y hacer de las mismas unas herramientas de trabajo efectivas, en beneficio de las actividades que se realizan [1-3]. Por lo tanto, la incursión de las Tecnologías de la Información y la Comunicación de carácter digital en la cotidianidad de los individuos, ha generado cambios en diferentes ámbitos de la sociedad: las relaciones interpersonales, forma, posibilidad de acceso al conocimiento, el acceso instantáneo a la amplia oferta de información online, las nuevas configuraciones económicas, políticas, el fenómeno de la globalización que cada vez se fortalece, toma más forma en la denominada sociedad de la información y la comunicación [3-5].

Una de las variables más importantes de las TIC's son los principios de la ejecución se basan en la planificación, la puesta en las prácticas de las acciones definidas en esa fase teórica es la que debe facilitar el logro de los objetivos que se hayan propuesto. Siendo lógico pensar la ejecución se debe hacer de la forma más próxima a la planificación y al diagnóstico se evidencio para luego habernos trazado el plan a aplicar [6,7]. Por ende, es pertinente establecer que La participación protagónica se basa en las estratégicas fundamentales que se dirigen en sentido de orientar las acciones de transformación a la realidad educativa como el adecuar el sistema educativo al modelo productivo, fortaleciendo e incentivando la investigación del proceso educativo desarrollando la educación sobre los proyectos productivos $[8,9]$.

En palabras de Padrón (2010) [10], señala que la participación protagónica surge de la creación en relación a la institución educativa, fundamentalmente logrando mantener la participación de los ciudadanos por la comunidad manteniendo organizado a los mismos en una entidad política, para activar el poder que considere necesario ordenar y exigir bajo la coordinación ejerciendo poder sobre un grupo de personas. Otro de los aspectos importantes, a destacar es la responsabilidad, esta conlleva a ocuparse de aquellas cuestiones que dependen más directamente del individuo. Por lo cual, el gerente debe proporcionar estrategias que estimulen al docente a realizar acciones que orienten el logro de las metas institucionales, desde la individualidad de sus funciones hacia el trabajo colectivo dentro de la institución educativa $[11,12]$.

En el mismo orden de ideas, Morgado et al. (2019) [13], exponen que el personal trabaja en una institución es responsable del buen desempeño de la misma. el gerente como líder de la institución al igual que los docentes tiene la responsabilidad de ejecutar sus funciones de una manera eficiente y eficaz, por cuanto dentro de su actuación debe predominar el sentido de compromiso hacia su gestión, así como el respeto hacia el cumplimiento de normas establecidas en la organización con el propósito del lograr una educación de calidad.

En relación a la corresponsabilidad de acuerdo con Terán et al. (2019) [14], manifiesta que implica que dos o más personas se hagan cargo material y/o moralmente, de las consecuencias de un hecho u omisión, provocado por ellos, por otros o por accidente; en forma conjunta, cooperativa y solidaria. Debido a todo lo expuesto en este trabajo se busca identificar los principios del uso de las TIC's para proyectos productivos en las instituciones educativas del Municipio Zona Bananera. Magdalena, Colombia. 


\section{Métodos y materiales}

El diseño de la presente investigación se considera como no experimental, transaccional y de campo. Con relación al diseño no experimental, Hernández et al. (2014) [15], lo definen como aquellos empleados en las investigaciones enfocadas a realizar descripciones de los fenómenos de estudio, pues las inferencias se realizan sin intervención directa o búsqueda de efectos en el fenómeno analizado y no se manipula la variable. En cuanto a la clasificación del diseño transaccional, le atribuyen la cualidad de ser "investigaciones que recopilan datos en un momento único"; razón por la cual no pretenden evaluar la evolución del comportamiento de la variable, sino que se enfocan en describir variables y analizar su interrelación en un momento dado. En lo que corresponde al diseño de campo, Arias (2012, p. 31) [16], señala que este "consiste en la recolección de datos directamente de los sujetos investigados, o de la realidad donde ocurren los hechos (datos primarios)".

\subsection{Población y muestra}

La población, de acuerdo con Oconnor et al. (2011) [17], es "el conjunto de todos los casos que concuerdan con determinadas especificaciones". Agregan los autores que un estudio no será mejor por tener mayor número de sujetos en su población lo importante es que ésta sea representativa e incluya las características necesarias para que el estudio sea confiable. En este sentido, la población objeto de estudio estará constituida por las instituciones de educación básica media del municipio Zona Bananera, tomando en cuenta los informantes cuya característica diferenciadora es su desempeño como directivos y docentes.

En cuanto a la muestra, como "un subgrupo de la población", no será necesario su cálculo pues se trabajará con el $100 \%$ de la población. La ejecución de la investigación, requiere como componente fundamental, la participación de sujetos que estén involucrados de modo directo con el objeto de estudio establecido; de allí, la importancia de precisarlos con el fin de tener claridad sobre el número de participantes que integran la población a ser consultados durante el proceso de recolección de información. En este sentido, Tinto (2013) [18], la definen como, la totalidad del fenómeno a estudiar, en donde las unidades de análisis poseen una característica común, la cual se estudia, dando origen a los datos de la investigación.

En este caso, la población estuvo conformada por directivos y docentes el cual sería abordado con doce (12) Instituciones Educativas de básica media del municipio rural Zona Bananera, departamento Magdalena - Colombia, donde se estudia a rectores y personal docente con relación a la problemática abordada.

\subsection{Técnicas e instrumentos de recolección de datos}

Según Díaz et al. (2013) [19], las técnicas consisten en las formas de recolectar la información a través de diferentes: encuestas, entrevistas, cuestionarios. Con relación a la presente investigación, se utilizó la técnica de observación por encuesta, por cuanto la información se recabó con el uso de un instrumento estructurado; dirigido a los rectores y docentes de las instituciones de básica media pertenecientes al municipio Zona Bananera del departamento del Magdalena-Colombia. Igualmente señala, que la aplicación de estos formularios supone que el investigador diseñe un cuestionario constituido por preguntas sobre determinados aspectos de la investigación; estos son referidos directamente a los indicadores que estructuran las bases teóricas del estudio.

Sobre la base de lo antes expuesto, el cuestionario para la indagación fue aplicado a rectores y docentes, el cuestionario quedó conformado por preguntas de tipo independiente elaboradas con tendencia de un 50\% de ítems positivos y un 50\% de preguntas negativas positiva y negativa, alternativas de respuestas cerradas y múltiples: TA (Totalmente de Acuerdo), PA (Parcialmente de Acuerdo), NA,ND (Ni de Acuerdo Ni en Desacuerdo), PD (Parcialmente en Desacuerdo) y TD (Totalmente en Desacuerdo), derivando las puntaciones 5, 4, 3, 2, 1 y viceversa. 


\subsection{Técnica de análisis de los datos}

La determinación de las técnicas de análisis, guarda relación con la selección de los medios y procedimientos a través de los cuales se extraen de los datos la información pertinente para elaborar respuestas a los cuestionamientos de la investigación; además, tal selección amerita mantener correspondencia entre las pruebas estadísticas y el tipo de investigación desarrollada. Es decir, que el análisis de los datos en este estudio, se realizó aplicando la estadística inferencial, concretamente el análisis de la varianza ANOVA de un factor de alternativas seleccionadas por los sujetos de la investigación en el instrumento de recolección de datos y posterior ubicación en baremo.

Al efecto, de acuerdo al criterio de Arias (2012) [16], se consideró el total de datos que resulta de multiplicar el número de sujetos por el número de ítems de cada indicador. Ahora bien, para la categorización de los valores presentados con la calificación, se realizó el siguiente baremo, el cual responde a la construcción siguiendo los métodos estadísticos para de esa manera determinar su ubicación. Así, para indicar las categorías en las cuales se ubican en la variable Uso de las TIC's para Proyectos productivos. de igual manera, se destaca que para la valoración de la media se estableció un baremo con el fin de categorizar los resultados. A continuación, en la Tabla 1, se presenta el mismo.

\begin{tabular}{ll}
\hline Categorías de Análisis & Rango \\
\hline Muy Alta Presencia & $4.21<5.00$ \\
Alta Presencia & $3.41<4.20$ \\
Mediana Presencia & $2.61<3.40$ \\
Poca Presencia & $1.81<2.60$ \\
Nula Presencia & $1.0<1.80$ \\
\hline
\end{tabular}

Table 1: Baremo de Categorías de Análisis y Rango.

\section{Resultados}

Se revelan los resultados referidos a los Principios del Uso de las TIC's, en el cual se muestra el comportamiento tanto de directores como docentes con relación a la ANOVA y la Prueba Post Hoc de Tukey.

\begin{tabular}{cccccc}
\hline & Suma de cuadrado & GI & Media cuadrática & F & Sig. \\
\hline Inter-grupos & 8,681 & 2 & 4,3420 & 9,064 & 0,000 \\
Intra-grupos & 202,562 & 423 &, 479 & & \\
$\quad$ total & 211,243 & 425 & & & \\
\hline
\end{tabular}

Table 2: ANOVA.

En la Tabla 2, se detalla el comportamiento de los encuestados, de allí que se aprecia el valor obtenido en la significancia, el cual es 0,000; puntaje menor que el valor referencial 0,005, indicativo de que existen diferencias altamente significativas entre los indicadores comparados, es decir, entre responsabilidad, corresponsabilidad y participación protagónica. Seguidamente, se especifica las medias arrojadas, así como las diferencias entre dichos indicadores.

La Tabla 3, exhibe los resultados de la prueba de Tukey, en esta se destaca el posicionamiento de cada uno de los indicadores que la conforman, los cuales se destacan en un ordenamiento diferente con relación a la fuente teórica consultada; secuencia mostrada en el cuadro de operacionalización de la variable. De allí, que las diferencias reveladas constituyeron dos subconjuntos, lo cual se debe a la variabilidad en las respuestas ofrecidas por los sujetos 


\begin{tabular}{c|ccc}
\hline $2^{*}$ Factor & $2^{*} \mathrm{~N}$ & \multicolumn{3}{c}{ Subconjunto para alfa $=0.05$} \\
\cline { 3 - 4 } & \multicolumn{3}{c}{1} \\
\hline Responsabilidad & 142 & 2,0728 \\
Corresponsabilidad & 142 & 2,1643 & 2,4108 \\
Participación protagónica & 142 & \multicolumn{3}{|c}{2,22} \\
Sig. & & 0,505 & 1,000 \\
\hline Media & \multicolumn{3}{|c}{} \\
\hline
\end{tabular}

Table 3: Prueba Post hoc de Tukey.

consultados. Con relación a los resultados derivados se observa que el valor más alto lo obtuvo fue Participación protagónica con 2,41, el cual se posesionó en el subconjunto dos y en la categoría de Poca presencia, seguido de Corresponsabilidad con 2,16 y Responsabilidad con 2,07, estos ubicados en el primer subconjunto y también en la categoría de Poca presencia. Finalmente, se destaca el valor de la dimensión; Principios del uso de las TIC's con una puntuación de 2,22, situada en la categoría de Poca presencia. Los resultados permiten inferir un comportamiento contrario al referido por Gámiz [20], quien expresa que los principios se basan en la planificación, la puesta en las prácticas de las acciones definidas en esa fase teórica es la que debe facilitar el logro de los objetivos que se hayan propuesto. Siendo lógico pensar la ejecución en el uso de las TIC's se debe hacer de la forma más próxima a la planificación y al diagnóstico, siendo favorable para luego haber trazado el plan aplicar, donde además los actores deben considerar para el abordaje de proyectos productivos ser responsables, corresponsables, pero además participar de manera protagónica.

Los hallazgos encontrados revelan que directivos y docentes con poca presencia identifican los principios del uso de las TIC's; Responsabilidad, corresponsabilidad y participación protagónica son considerados en las instituciones educativas para elevar la capacidad productiva en el desarrollo de proyectos productivos. De allí, que todas las acciones emprendidas resultarán desfavorables en la iniciativa de elevar la calidad de la educación y los procesos escolares en el Municipio de la Zona Bananera.

Las revelaciones acontecidas muestran ineficiencia en directivos y docentes para el desarrollo de proyectos productivos, puesto que los principios no son aplicados para desarrollar la planificación educativa centrada en el aspecto endógeno, no siendo favorable para dar respuestas a necesidades internas y al sistema productivo de la institución educativa con el abordaje de la tecnología. De allí, que los procesos gestados en los centros poco se orientan a convertir las Tecnologías de Información y Comunicaciones en un mecanismo de participación, construcción del conocimiento, ni tampoco se ofrecen oportunidades de crecimiento responsable por parte de estas personas.

\section{Conclusiones}

Los directivos y docentes con poca presencia identifican los principios del uso de las TIC's; Responsabilidad, corresponsabilidad y participación protagónica, por lo cual no son considerados en las instituciones educativas para elevar la capacidad productiva en el desarrollo de proyectos productivos. De allí, que todas las acciones emprendidas resultarán desfavorables en la iniciativa de elevar la calidad de la educación y los procesos escolares en el Municipio. Se hace necesario promover la reorientación de la gestión de la filosofía institución hacia la construcción de experiencias significativas con el desarrollo de proyectos productivos impulsados desde la gerencia al aula de clase, donde oportunamente cada docente renovará sus acciones en aras de mejorar la responsabilidad, corresponsabilidad y su participación en cada de las actividades asociadas con las TIC's. En este sentido, las reuniones, círculos de formación constructiva y encuentros de 
pares, fomentarán una cultura basada en la moral, ética y responsabilidad hacia cada uno de los proyectos impulsados con orientación endógena, a bien de la obtención de beneficios económicos.

\section{References}

[1] K. Wheatley, D. Arroyo, J. Quiñónez, J. Moreira, M. Wila, D. González, "Sistema de Información Web para la gestión solicitudes de Jornadas Sociales en Telecomunicaciones por parte de organizaciones públicas y privadas," Revista Arbitrada Interdisciplinaria Koinonía, vol. 4, no. 8, pp. 592-622, 2019.

[2] C. Latta, "Uso de las TIC para proyectos productivos en las instituciones educativas del Municipio Zona Bananera. Magdalena. Colombia," Revista Arbitrada Interdisciplinaria Koinonía, vol. 4, no. 7, pp. 233-246, 2019.

[3] M. Moreno, L. Montoya, "Nuevas interacciones sociales: Formas de entender el flujo de la comunicación en las organizaciones y la cotidianidad," Observatorio, vol. 9, no. 1, pp. 131-148, 2015.

[4] J. Sánchez, M. González, M. Muñoz, "La sociedad de la información: génesis, iniciativas, concepto y su relación con las TIC," Revista UIS Ingenierías, vol. 11, no. 1, pp. 113-128, 2012.

[5] J. López, "El proceso de capacitación, sus etapas e implementación para mejorar el desempeño del recurso humano en las organizaciones," Contribuciones a la Economía, vol. 12, pp. 1-18, 2011.

[6] V. Reyes, C. Reading, H. Doyle, S. Gregory, "Integrating ICT into teacher education programs from a TPACK perspective: Exploring perceptions of university lecturers," Computers Education, vol. 115, pp. 1-19, 2017.

[7] M. Rodríguez, "Indicadores de gestión en la gerencia estratégica universitaria," Orbis. Revista Científica Ciencias Humanas, vol. 9, no. 27, pp. 31-46, 2014.

[8] M. Butter, L. Jiménez, M. Badilla, "School networks to promote ICT competences among teachers. Case study in intercultural schools," Computers in Human Behavior, vol.30, pp. 442-451, 2014.

[9] K. Holden, A. Van Klyton, "Exploring the tensions and incongruities of Internet governance in Africa," Government Information Quarterly, vol. 33, no. 4, 2016.

[10] G. Padrón, "La participación protagónica estudiantil en el proceso de extensión universitaria," Revista Latinoamericana de Ciencias Sociales, Niñez y Juventud, vol. 8, no.1, pp. 347-362, 2010.

[11] A. García, M. Ruiz, C. Severiche, M. Martínez, "Tipos de conocimiento para procesos administrativos: el caso de los Centros Universitarios del Departamento del Atlántico Norte de Colombia," En Meriño, V., Martinez, E., Martínez, C. (Ed.). Gestión del Conocimiento. Perspectiva Multidisciplinaria. Santa Bárbara - Zulia - Venezuela: Fondo Editorial Universitario de la Universidad Nacional Experimental del Sur del Lago de Maracaibo Jesús María Semprún, vol. 16, pp. 289-300, 2019.

[12] C. Severiche, D. Muñoz, J. Jaimes, "Gestión del conocimiento en sector de agua potable y saneamiento básico en Colombia," Revista Omnia, vol. 22, no. 1, pp. 91-105, 2016.

[13] C. Morgado, J. Zavala, J. Argüelles, "Gerencia transformacional desde el accionar docente directivo para el abordaje del Contexto Escolar," CIENCIAMATRIA, vol. 5, no. 9, pp. 48-72, 2019.

[14] A. Terán, G. Dávila, R. Castañón, "Gestión de la tecnología e innovación: un Modelo de Redes Bayesianas". Economía: Teoría y Práctica, vol. 50, pp. 63-100, 2019.

[15] O. Hernández, H. Jurado, Y. Romero, "Análisis de publicaciones hispanoamericanas sobre TIC en escuelas y zonas rurales," Revista Colombiana de Educación, vol. 66, pp. 103-126, 2014.

[16] F. Arias, "El proyecto de Investigación. Introducción a la metodología científica," Editorial Episteme. 5ta Edición, 2012.

[17] L. Oconnor, A. Zaldívar, E. Hernández, "Un acercamiento al estudio de la integración de métodos teóricos de la investigación científica,". Revista Universitaria Arbitrada de Investigación y Diálogo Académico, vol. 7, no. 1, pp. 142-57, 2011.

[18] J. Tinto, "El análisis de contenido como herramienta de utilidad para la realización de una investigación descriptiva. Un ejemplo de aplicación práctica utilizado para conocer las investigaciones realizadas sobre la imagen de marca de España y el efecto país de origen," Provincia, vol. 29, no. 1, pp. 135-173, 2013.

[19] L. Díaz, U. Torruco, M. Martínez, M. Varela, "La entrevista, recurso flexible y dinámico. Investigación en Educación Médica," vol. 2, no. 7, pp. 162-167, 2013.

[20] V. Gámiz, "ICT-based Active Methodologies," Procedia - Social and Behavioral Sciences, vol. 237, pp. 606-612, 2017.
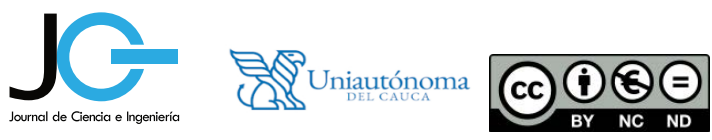